\title{
多官能团羰基化合物的高选择性不对称氢化
}

\author{
马 欣 ${ }^{a}$ 李万方 ${ }^{a}$ 范为正 ${ }^{a}$ 陶晓明 ${ }^{a}$ 李晓明 ${ }^{a}$ 姚 莹 ${ }^{a}$ \\ 诸吕锋 ${ }^{b}$ 陈厚和 ${ }^{b}$ 谢小敏 ${ }^{a}$ 张兆国*,a,c \\ $\left({ }^{a}\right.$ 上海交通大学化学化工学院 上海 200240) \\ ( ${ }^{b}$ 南京理工大学化工学院 南京 210094) \\ ( ${ }^{c}$ 中国科学院上海有机化学研究所 金属有机化学国家重点实验室 上海 200032)
}

\begin{abstract}
摘要 简要地介绍了我们小组最近在多官能团化羰基底物的区域和立体选择性氢化方面的一些进展, 对该研究领域的 继续深入应用前景进行了展望.

关键词 不对称氢化; 嶡基; 多官能团化; 化学选择性; 对映选择性
\end{abstract}

\section{Study on the Highly Selective Asymmetric Hydrogenation of Polyfunctionalized Carbonyl Substrates}

\author{
$\mathrm{Ma}, \mathrm{Xin}^{a} \quad \mathrm{Li}$, Wanfang ${ }^{a}$ \\ Fan, Weizheng ${ }^{a}$ \\ Tao, Xiaoming ${ }^{a}$ \\ Li, Xiaoming ${ }^{a}$ \\ Yao, Ying $^{a} \quad$ Zhu, Lüfeng ${ }^{b}$ \\ Chen, Houhe ${ }^{b}$ \\ Xie, Xiaomin ${ }^{a}$ \\ Zhang, Zhaoguo ${ }^{*, a, c}$ \\ $\left({ }^{a}\right.$ School of Chemistry and Chemical Engineering, Shanghai Jiao Tong University, Shanghai 200240) \\ $\left({ }^{b}\right.$ School of Chemical Engineering, Nanjing University of Science \& Technology, Nanjing 210094) \\ ( ${ }^{c}$ State Key Laboratory of Organometallic Chemistry, Shanghai Institute of Organic Chemistry, \\ Chinese Academy of Sciences, Shanghai 200032)
}

\begin{abstract}
In this account, our recent progress in highly chemo- and stereo-selective asymmetric hydrogenation of some polyfunctionalized carbonyl substrates is briefly reviewed. The furtherance of the study in this field and their applications is prospected.

Keywords asymmetric hydrogenation; carbonyls; polyfunctionalized; chemoselectivity; enantioselectivity
\end{abstract}

不对称氢化在手性合成领域占有十分重要的地位, 其中羰基化合物的不对称氢化是构建手性仲醇的一种 有效的方法 ${ }^{[1]}$. Noyori 等 ${ }^{[2]}$ 发展的 BINAP 配体, 使得羰 基化合物的不对称氢化在有机合成中的应用越来越广 泛; 在此基础上发展出来的 SegPhos 系列配体, 是一个 比 BINAP 更有效的配体. 迄今为止, 许多以联芳基为 骨架的配体被设计合成出来 ${ }^{[3]}$ (Scheme 1), 在官能团化 的羰基化合物的氢化中取得了优异的结果.

到目前为止，大多数单官能团羰基化合物(羰基临 近只有单个导向基团)和简单酮(羰基周围没有导向基 团)的不对称氢化已经被很好地解决. ${ }^{[4]}$ 但是当羰基邻

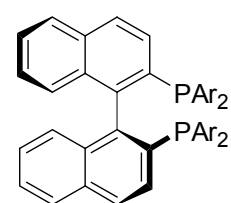

(S)-BINAP

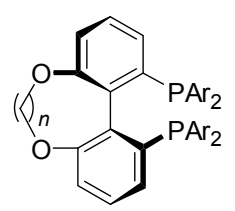

(S)-C -TunePhos<smiles>Cc1cccc(P)c1-c1c(C)cccc1P(=O)(O)O</smiles>

(S)-BIPHEMP<smiles>O=[PH](=O)(O)c1ccc2c(c1-c1c(P)ccc3c1OCO3)OCO2</smiles>

(S)-SEGPhos

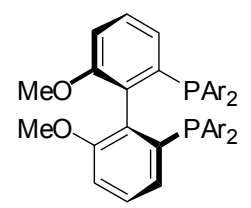

(S)-MeO-BIPHEP<smiles>CC1(C)Oc2ccc(P)c(-c3c([PH](#N)(F)F)ccc4c3OC(C)(C)O4)c2O1</smiles>

(S)-SunPhos

Scheme 1

*E-mail: zhaoguo@sjtu.edu.cn

Received May 7, 2012; revised June 18, 2012; published online June 20, 2012.

Project supported by the National Natural Science Foundation of China (Nos. 20872090, 21172146).

国家自然科学基金(Nos. 20872090, 21172146)资助项目. 
近存在有两个或多个官能团时，这些底物的不对称氢化 变得比较困难: 多个导向基团参与的竞争配位 ${ }^{[5]}$, 使得 产物的对映选择性不理想; 尤其是当这些官能团的导 向能力接近的情况下, 氢化反应的对映选择性往往较 低. 这是因为, 对于羰基的周围有两个配位能力相近的 基团时, 两种可能的配位模式都存在, 如果其中一种配 位模式导致 $R$-构型的氢化产物, 那么另一种配位模式 将导致 $S$-构型的氢化产物. 理论上讲, 如果两种配位模 式的差别不是足够大, 将不可能获得高的对映选择性 (Scheme 2).

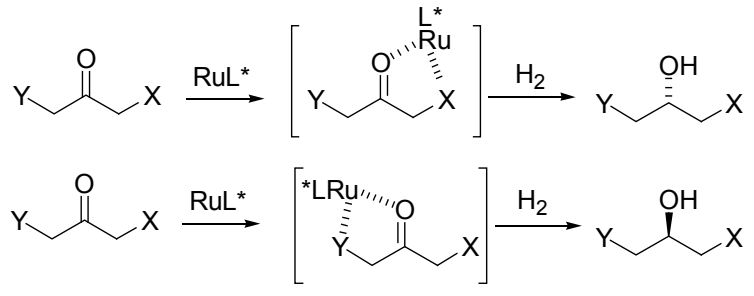

Scheme 2

我们小组 ${ }^{[6]}$ 设计合成了 SunPhos 系列配体, 并利用 该配体实现了对一系列单官能团酮的不对称氢化 ${ }^{[7]}$. 最 近几年我们对更有挑战性的多官能团羰基化合物的不 对称氢化进行了研究, 本文主要对我们小组最近在这方 面的研究工作进行总结.

\section{4-芳基-2-氧代-3-丁烯酸乙酯的不对称氢化}

2-羟基-4-苯基丁酸酯是一个非常重要的手性中间 体, 是合成普利类药物的关键原料. 已报道的不对称氢 化是以 4-苯基-2-氧代丁酸乙酯为原料来实现的 ${ }^{[8]}$, 但因 为原料的稳定性差, 反应必须使用新纯化的原料, 否则 反应难以重复. 我们用苯甲醛和丙酮酸乙酯合成了 4-苯 基-2-氧代丁烯酸乙酯, 该化合物化学性质远比 4-苯基2-氧代丁酸乙酯稳定, 而且合成方法简洁, 产率高, 易 于大规模合成. 对于这样一个反应物, 它不但在羰基的 邻位有一个酯基作为配位基团, 还有一个碳-碳双键同 时可以作为配位基团. 我们以 $\left[\mathrm{NH}_{2} \mathrm{Me}_{2}\right]^{+}\{[\mathrm{RuCl}(S)-$ SunPhos $\left.]_{2}\left(\mu-\mathrm{Cl}_{3}\right)\right\}$ 为催化剂, 通过加入 6 equiv. 于催化剂 的 $1 \mathrm{~mol} \cdot \mathrm{L}^{-1}$ 盐酸, 以 $94 \%$ 的 $e e$ 值得到了双键和羰基均 被还原的产物 4-苯基-2-羟基丁酸乙酯. 其他的 4-芳基2-氧代丁烯酸乙酯均能以较高的对映选择性( $94 \%$ $96 \%$ ee) 得到氢化产物(Table 1). 当把反应物和催化剂的 物质的量比提高到 2000 时, 反应的 $e e$ 值没有明显降低, 仍然达到 93\%. 产物经过水解、一次重结晶、酯化后可 以得到 $e e$ 值高达 $99 \%$ 的 4-苯基-2-羟基-3-丁酸乙酯. 进一 步的研究表明, 不对称氢化反应是羰基先被还原, 碳一碳 双键再进一步被还原的 ${ }^{[9]}$.
表 1 4-芳基-2-氧代-3-丁烯酸乙酯的不对称氢化 ${ }^{a}$

Table 1 Asymmetric hydrogenation of ethyl 4-aryl-2-oxo3-butenoate

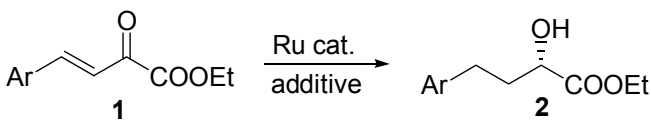

\begin{tabular}{cclc}
\hline Entry & $\mathbf{1}$ & \multicolumn{1}{c}{$\mathrm{Ar}$} & $e e^{b} / \%$ \\
\hline 1 & $\mathbf{1 a}$ & $\mathrm{C}_{6} \mathrm{H}_{5}$ & 94 \\
2 & $\mathbf{1 b}$ & $4-\mathrm{Me}-\mathrm{C}_{6} \mathrm{H}_{4}$ & 95 \\
3 & $\mathbf{1 c}$ & $4-\mathrm{F}-\mathrm{C}_{6} \mathrm{H}_{4}$ & 95 \\
4 & $\mathbf{1 d}$ & $4-\mathrm{Cl}-\mathrm{C}_{6} \mathrm{H}_{4}$ & 95 \\
5 & $\mathbf{1 e}$ & $4-\mathrm{Br}-\mathrm{C}_{6} \mathrm{H}_{4}$ & 96 \\
6 & $\mathbf{1 f}$ & $4-\mathrm{MeO}_{4}-\mathrm{C}_{6} \mathrm{H}_{4}$ & 95 \\
7 & $\mathbf{1 g}$ & $3-\mathrm{Cl}_{6}-\mathrm{C}_{6} \mathrm{H}_{4}$ & 95 \\
8 & $\mathbf{1 h}$ & $2,4-\mathrm{Cl}_{2}-\mathrm{C}_{6} \mathrm{H}_{5}$ & 95 \\
9 & $\mathbf{1 i}$ & Benzo $[d][1,3]$ dioxol-5-yl & 95 \\
\hline
\end{tabular}

${ }^{a}$ All reactions were carried out under 1.4 MPa of hydrogen with a substrate (1 mmol) concentration of $0.2 \mathrm{~mol} \cdot \mathrm{L}^{-1}$ in $\mathrm{EtOH}$ at $70{ }^{\circ} \mathrm{C}$ for $12 \mathrm{~h}$. Substrate : catalyst $: 1 \mathrm{~mol} \cdot \mathrm{L}^{-1} \mathrm{HCl}=100: 1: 6$ (molar ratio), conversion $100 \%{ }^{b}{ }^{b} e e$ values were determined by HPLC analysis.

为了得到光学纯的 2-羟基-4-苯基丁酸酯, 上述氢 化产物必须经过水解、重结晶、再酯化等化学转换过程. 我们于是尝试了 2-氧代-4-苯基-3-丁烯酸的直接不对称 氢化. $\alpha$-羰基羧酸的直接不对称氢化一直是一个难以解 决的问题，因为生成的产物 $\alpha$-羟基羧酸是一个配位能力 比原料 $\alpha$-羰基羧酸更强的配体，会导致催化剂中毒，不 对称催化将难以进行. 通过优化反应条件，我们实现了 2-氧代-4-苯基-3-丁烯酸及其衍生物的不对称氢化，简 化了反应步骤. 对该反应深入研究发现, 2-氧代- 4-苯基 丁酸的不对称氢化速度和 $e e$ 值都优于 2-氧代- 4-苯基-3丁烯酸的不对称氢化结果. 在此基础上，我们还实现了 对应的饱和化合物 2-氧代-4-芳基丁酸(3)的直接氢化还 原, 也得到较好的结果(Eq. 1 ${ }^{[10]}$.

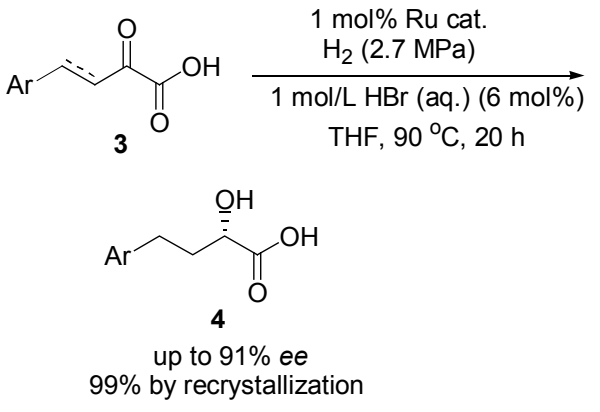

\section{$2 \gamma$-杂原子取代的 $\beta$ 酮酸酯的不对称氢化}

Ru-BINAP 体系催化的不对称氢化简单的 $\beta$-酩酸酯 已经取得了较好的成果, 以及后来发展的 $\mathrm{Ru}$-轴手性的 联芳基手性双膦配体催化体系对 $\beta$-酮酸酯的不对称氢 化也有相当或者更好的结果. 但是对于那些邻近含有杂 
原子以及多官能化 $\beta$-酮酸酯的不对称氢化研究却比较 少. 由于邻近配位基团与催化剂发生竞争配位使得催化 剂和酯羰基的配位有所削弱而导致对映选择性大幅降 低, 仅有少数邻近含有杂原子或者多官能化的 $\beta$-酮酸酯 例子, 如邻近含有芐氧基、烷氧基、卤素、氮以及碳碳双键. 最近, 我们小组实现了一系列 $\gamma$-杂原子取代的 $\beta$-酮酸酯的不对称氢化 ${ }^{[11]}$. 对于配位能力较弱的氯原子 和氧原子, 仅仅通过提高反应温度, 就能够取得较好的 不对称催化效果; 对于配位能力较强的硫原子, 我们将 其氧化成高价态的砜, 削弱了其配位能力, 取得了 98.2\%的对映选择性; 对于 $\mathrm{N}$ 原子，我们用吸电子基团 保护基，降低其参与和催化剂金属中心竞争配位的能 力, 取得了 $98.0 \%$ 的对映选择性(Table 2).

表 $2 \gamma$-杂原子取代的 $\beta$-酮酸酯的不对称氢化 ${ }^{a}$

Table 2 Asymmetric hydrogenation of $\beta$-ketoesters with a $\gamma$-coordinating substituent

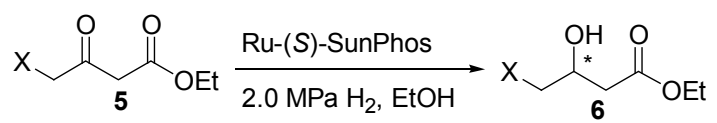

\begin{tabular}{cclc}
\hline Entry & $\mathbf{5}$ & \multicolumn{1}{c}{$\mathrm{X}$} & $e e^{b} / \%$ \\
\hline 1 & $\mathbf{5 a}$ & $\mathrm{BnO}$ & 99.1 \\
2 & $\mathbf{5 b}$ & $\mathrm{Cl}$ & 96.5 \\
3 & $\mathbf{5 c}$ & $\mathrm{AcO}$ & 99.0 \\
4 & $\mathbf{5 d}$ & $\mathrm{PhSO}_{2}$ & 98.2 \\
5 & $\mathbf{5 e}$ & $\mathrm{BocNH}$ & 98.0 \\
6 & $\mathbf{5 f}$ & $\mathrm{CbzNH}$ & 97.2 \\
\hline
\end{tabular}

${ }^{a}$ All reactions were carried out with a substrate $(1.0 \mathrm{mmol})$ concentration of $0.4 \mathrm{~mol} \cdot \mathrm{L}^{-1}$ in EtOH under $2.0 \mathrm{MPa}$ of $\mathrm{H}_{2}$ at $70{ }^{\circ} \mathrm{C}$ for $18 \mathrm{~h}$. Sustrate/ catalyst $=100 / 1$ (molar ratio). Conversion $=100 \%{ }^{b}$ ee values were determined by HPLC.

这些含有 $\gamma$-配位取代基的原料的氢化产物，在有机 合成方面具有广泛的应用 ${ }^{[12]}$. 我们将 $\mathbf{5 g}$ 进行不对称氢 化 ${ }^{[13]}$, 得到了 $97.1 \% e e$ 的氢化产物, 经过几步常规的转 化, 我们得到了 $98 \%$ ee 的化合物 $\mathbf{6 g}$ (Scheme 3), 它可 以作为抗菌药物 Sitafloxacin 和 Olamufloxacin 的合成关 键中间体.

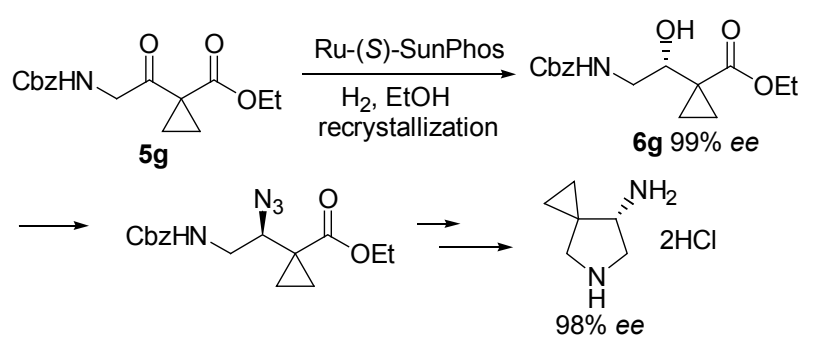

Scheme 3

对于 $\beta, \delta$-双氧代羒酸酯的不对称氢化,一直是一个 悬而未决的难题. 不论是对映选择性, 还是非对映选择
性，都不能得到有效解决，而且该类反应物的活性也不 高 ${ }^{[14]}$. 为了解决这一问题, 我们首先采取了比较保守的 方法，首先将 $\delta$-羰基保护，然后再进行不对称氢化. 5羰基乙二醇保护的 3,5-二羰基羧酸酯(7)的不对称氢化 可以高效进行，产物 8 脱保护后，可以再经过底物控制 的硼氢化物还原得到高光学纯度的 3,5-二羟基羧酸酯异 构体(Table 3) ${ }^{[15]}$.

\section{表 $3 \delta$-缩酮- $\beta$-酮酸酯的不对称氢化 ${ }^{a}$}

Table 3 Asymmetric hydrogenation of ethylene glycol-protected-dioxocarboxylic esters
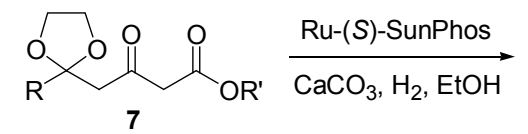

$\mathrm{R}=\mathrm{ClCH}_{2}, \mathrm{Me}, \mathrm{Et}, \mathrm{Ar}$, etc.

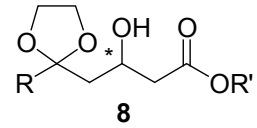

up to $99 \%$ ee

\begin{tabular}{cclccc}
\hline Entry & $\mathbf{7}$ & $\mathrm{R}$ & $\mathrm{R}^{\prime}$ & $e e / \%$ \\
\hline 1 & $\mathbf{7 a}$ & $\mathrm{ClCH}_{2}$ & $\mathrm{Me}$ & $99.7^{b}$ \\
2 & $\mathbf{7 b}$ & $\mathrm{ClCH}_{2}$ & $\mathrm{Et}$ & $95.7^{b}$ \\
$3^{c}$ & $\mathbf{7 c}$ & $\mathrm{OBn}$ & $\mathrm{Me}$ & $99.2^{d}$ \\
$4^{e}$ & $\mathbf{7 d}$ & $\mathrm{OBn}$ & $\mathrm{Et}$ & $99.2^{d}$ \\
5 & $\mathbf{7 e}$ & $\mathrm{OBn}$ & $t-\mathrm{Bu}$ & $99.6^{d}$ \\
6 & $\mathbf{7 f}$ & $\mathrm{Me}$ & $\mathrm{Me}$ & $99.3^{b}$ \\
7 & $\mathbf{7 g}$ & $\mathrm{Et}$ & $\mathrm{Me}$ & $99.5^{b}$ \\
\hline
\end{tabular}

${ }^{a}$ Unless otherwise specified. All reactions were carried out with a substrate $(1.25 \mathrm{mmol})$ concentration of $0.4 \mathrm{~mol} \cdot \mathrm{L}^{-1}$ in $\mathrm{EtOH}, \mathrm{CaCO}_{3}(12 \mathrm{mg})$ as additive, under 6.0 MPa of $\mathrm{H}_{2}$ at $55{ }^{\circ} \mathrm{C}$ for $4.5 \mathrm{~h}$. Sustrate/catalyst $=250 / 1$ (molar ratio). ${ }^{b} e e$ values of their $p$-nitrobenzoates. ${ }^{c} 65{ }^{\circ} \mathrm{C} .{ }^{d} e e$ values were determined directly by HPLC. ${ }^{e} 75{ }^{\circ} \mathrm{C}, 10 \mathrm{~h}$.

\section{$3 \gamma$-卤代- $y, \delta$-不饱和- $\beta$-酮酸酯}

手性的仲烯丙醇是一类重要的合成中间体，不仅在 许多天然产物和药物分子中广泛存在，而且可以通过许 多化学转化, 实现手性的传递, 如 Claisen 重排 ${ }^{[16]}$ 或者 $\mathrm{S}_{\mathrm{N}} 2^{\prime}$ 取代反应 ${ }^{[17]}$ 等. 由 $\alpha, \beta$-不饱和酮不对称还原是制备 手性仲烯丙醇的重要方法之一. 手性铝试剂 ${ }^{[18]}$ 、嗍试 剂[19]还原需要化学计量的还原剂, Noyori 小组 ${ }^{[20]}$ 发展起 来的 $\mathrm{Ru} /$ 双膦/双胺体系应用于 $\alpha, \beta$-不饱和酮还原时获得 了很好的化学选择性和对映选择性, 但此催化体系却不 适用于 $\beta$-酮酸酯底物 ${ }^{[21]}$.

最近我们 ${ }^{[22]}$ 实现了酮羰基附近含有共轭双键和卤 素的高度官能团化的 $\beta$-酮酸酯的选择性羰基不对称氢 化，取得了较高的化学选择性和对映选择性. 通过调控 反应条件，使得通常在氢化条件下敏感的共轭烯烃双键 和烯基卤原子都得到了保留(Table 4). 这些多官能化的 产物, 可以进行进一步的化学转化, 合成结构更为复杂 的具有生物活性和特种功能的分子.

而且烯基溴可以很方便地在钯催化下脱除，且没有 发生消旋，得到含有反式烯丙醇和 $\beta$-羊基醇单元的化合 物 11, 可以用于多种他汀侧链的合成(Eq. 2). 
表 $4 \gamma$-卤代- $\gamma, \delta$-不饱和- $\beta$-酮酸酯的不对称氢化 ${ }^{a}$

Table 4 Asymmetric hydrogenation of 5-aryl-4-halo-3-oxo4-pentenoates

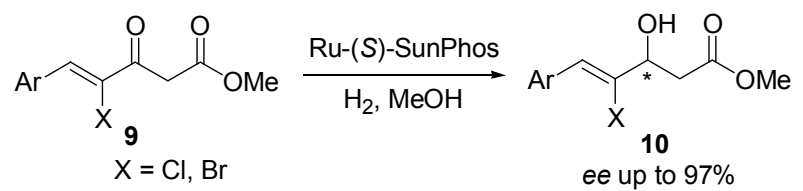

\begin{tabular}{cclccc}
\hline Entry & $\mathbf{9}$ & $\mathrm{Ar}$ & $\mathrm{X}$ & $\mathrm{Yield}^{b} / \%$ & $e e^{c} / \%$ \\
\hline 1 & $\mathbf{9 a}$ & $\mathrm{C}_{6} \mathrm{H}_{5}$ & $\mathrm{Cl}$ & 96 & 95.7 \\
2 & $\mathbf{9 b}$ & $4-\mathrm{Me}-\mathrm{C}_{6} \mathrm{H}_{4}$ & $\mathrm{Cl}$ & 98 & 96.0 \\
3 & $\mathbf{9 c}$ & $4-\mathrm{MeO}-\mathrm{C}_{6} \mathrm{H}_{4}$ & $\mathrm{Cl}$ & 98 & 95.6 \\
4 & $\mathbf{9 d}$ & $2-\mathrm{MeO}-\mathrm{C}_{6} \mathrm{H}_{4}$ & $\mathrm{Cl}$ & 98 & 97.0 \\
5 & $\mathbf{9 e}$ & $4-\mathrm{Br}-\mathrm{C}_{6} \mathrm{H}_{4}$ & $\mathrm{Cl}$ & 96 & 95.1 \\
6 & $\mathbf{9 f}$ & $4-\mathrm{CF}_{3}-\mathrm{C}_{6} \mathrm{H}_{4}$ & $\mathrm{Cl}$ & 95 & 94.9 \\
7 & $\mathbf{9 g}$ & $E-\mathrm{PhCH}_{2}=\mathrm{CH}$ & $\mathrm{Cl}$ & 97 & 94.1 \\
7 & $\mathbf{9 h}$ & $\mathrm{C}_{6} \mathrm{H}_{5}$ & $\mathrm{Br}$ & 92 & 96.3 \\
$8^{d}$ & $\mathbf{9 i}$ & $4-\mathrm{MeO}_{2} \mathrm{C}_{6} \mathrm{H}_{4}$ & $\mathrm{Br}$ & 91 & 96.4 \\
$9^{e}$ & $\mathbf{9 j}$ & $4-\mathrm{CF}_{3}-\mathrm{C}_{6} \mathrm{H}_{4}$ & $\mathrm{Br}$ & 92 & 95.4 \\
\hline
\end{tabular}

${ }^{a}$ All reactions were carried out with a substrate $(1.0 \mathrm{mmol})$ concentration of $0.5 \mathrm{~mol} \cdot \mathrm{L}^{-1}$ in $\mathrm{MeOH}$ at $70{ }^{\circ} \mathrm{C}$ for $0.5 \mathrm{~h}$ under $1.0 \mathrm{MPa}$ of $\mathrm{H}_{2}$, Sustrate/catalyst $=200 / 1$ (molar ratio). ${ }^{b}$ Isolated yield. ${ }^{c} e e$ values were determined by HPLC on a Chiralpak IC-3 column. ${ }^{d} 70 \mathrm{~min} .{ }^{e} 100 \mathrm{~min}$,
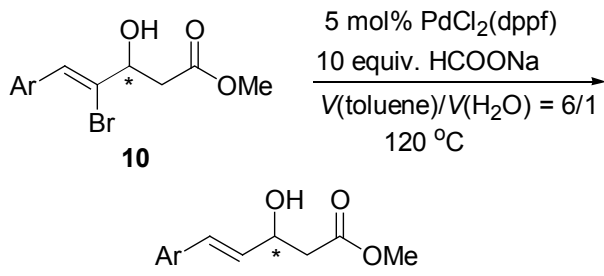

11

\section{4 多羰基酮的选择性不对称氢化}

羰基邻近有多个性质相近的导向基团存在时，往往 不能取得好的立体选择性(例如当 $\mathrm{X}=\mathrm{COOEt}, \mathrm{Y}=$ COOBu- $t$ 时, 在 Ru-SunPhos 体系下, 我们只得到了 $14.7 \%$ 的对映选择性). 这主要是因为它们都会参与导 向, 而且两种类似的导向基团起主要导向作用时, 产物 构型正好相反. 我们通过实验的方法测试了常见的官能 才的导向能力的强弱顺序, 特别是对于同一类型的导向 基团, 希望找到影响它们导向能力的具体因素, 这能够 为调控这类底物的高选择性不对称氢化提供理论依据.

\subsection{3 -氧代戊二酸衍生物的不对称氢化}

为了得到羧酸衍生物羰基的配位能力顺序, 我 们 ${ }^{[23]}$ 比较了不同的羧酸衍生物氢化反应速率(Eq. 3)

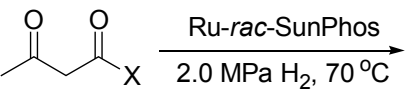

$$
\begin{aligned}
& 12 \mathrm{a} \sim 13 \mathrm{j}
\end{aligned}
$$

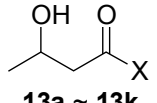

$$
13 a \sim 13 k
$$

12i $X=$ morpholyl

12j $\mathrm{X}=\mathrm{NH} t-\mathrm{Bu}$

$12 \mathbf{k}=\mathrm{NHPh}$
在乙醇体系中，我们可得出如下结论：叔丁酯 $12 \mathrm{c}$ 略快于其它酯，但彼此之间差别不大；叔丁胺 $\mathbf{1 2 j}$ 快于 乙酯 12a, 差别较大; 乙酰芳胺的最慢, 仲酰胺的最快, 但彼此差别都不大.

在混合实验比较速率的过程中发现，当混合组分中 含有 $12 f$ 和 $12 i$ 时，整个体系总是不能完全转化，而且变 得杂乱. 分析发现，由于底物 $12 \mathrm{f}$ 和 $12 \mathrm{i}$ 在该反应体系中 发生了醇解生成大量的 $12 \mathrm{a}$, 因而有游离的仲胺释放出 来使催化剂中毒. 为了避免醇解, 我们使用 THF 作为溶 剂, 不仅避免了醇解, 而且发现在 THF 中的反应速率关 系: 各种 $\beta$-酮酸酯基本都不反应，各种 $\beta$-酥酰胺反应较 快. 基于此, 我们实现了 2-氧代戊二酸衍生物在 THF 中 的该选择性不对称氢化.

表 53 -氧代戊二酸衍生物的不对称氢化 ${ }^{a}$

Table 5 Asymmetric hydrogenation of $\mathbf{1 4}$

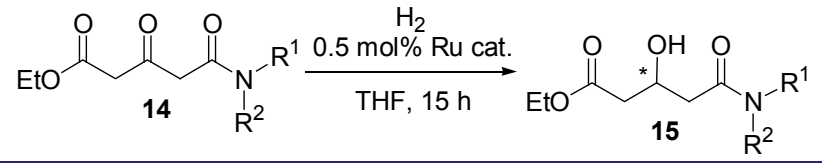

\begin{tabular}{cclcc}
\hline Entry & $\mathbf{1 4}$ & \multicolumn{1}{c}{$\mathrm{NR}^{1} \mathrm{R}^{2}$} & $e e / \%$ in THF & $e e / \%$ in $\mathrm{EtOH}$ \\
\hline 1 & $\mathbf{1 4 a}$ & $\mathrm{NEt}_{2}$ & 96.5 & 93.4 \\
2 & $\mathbf{1 4 b}$ & $\left(\mathrm{CH}_{2}\right)_{4}$ & 96.7 & 81.7 \\
3 & $\mathbf{1 4 c}$ & $\left(\mathrm{CH}_{2}\right)_{2} \mathrm{O}\left(\mathrm{CH}_{2}\right)_{2}$ & 96.4 & $\mathbf{N}^{b} \mathbf{A}^{b}$ \\
4 & $\mathbf{1 4 d}$ & $\mathrm{NPh}_{2}$ & 98.1 & 53.3 \\
5 & $\mathbf{1 4 e}$ & $\mathrm{NMeO}$ & 87.3 & $\mathbf{N}^{b} \mathbf{A}^{b}$ \\
6 & $\mathbf{1 4 f}$ & $\mathrm{NH} t-\mathrm{Bu}$ & $94.4^{c}$ & $91.4^{c}$ \\
7 & $\mathbf{1 4 g}$ & $\mathrm{NHBn}$ & 87.1 & 79.3 \\
\hline
\end{tabular}

${ }^{a}$ All reactions were carried out in THF or in EtOH with a substrate $(1 \mathrm{mmol})$ concentration of $0.2 \mathrm{~mol} \cdot \mathrm{L}^{-1}$ at $70{ }^{\circ} \mathrm{C}$ with $2.0 \mathrm{MPa}$ of $\mathrm{H}_{2}$ for $15 \mathrm{~h}$. Substrate/catalyst $=200 / 1$ (molar ratio). Conversions were $100 \%$ except where indicated. $e e$ 's were determined on HPLC. ${ }^{b}$ No product was obtained due to alcoholysis of the substrate. ${ }^{c} e e$ of its 4-nitrobenzoate.

在同一分子中有两个 $\beta$-酮酸衍生物体系，选择性氢 化其中的一个酮羰基是困难的，之前也有人进行了研 究，但是一直没有解决. 我们利用在 THF 这样一种不常 用的反应溶剂中 $\beta$-酮酸酯和酮酰胺截然相反的反应活 性实现了这一目的. 对于化合物 16, 在 Ru-SunPhosTHF 体系中我们实现了高选择性地实现 8-位羰基的氢 化, ee 值高达 97.7\% (Eq. 4).

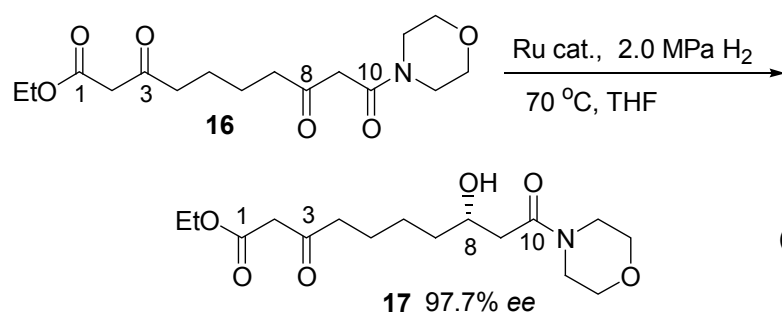

\section{$4.23,5-$ 二氧代羧酸衍生物的不对称氢化}

光学纯的 3,5-二羟基羧酸衍生物结构单元在天然产 
物和药物分子中大量存在, 但目前合成这类化合物并没 有特别高效的办法. 5-位着基的产物可以通过 Aldol 反 应得到, 最近发展的生物催化的方法在这方面也取得了 一些进展, 但有较多的局限性. 20 世纪 90 年代, 有一些 课题组开始研究 3,5-二氧代羧酸酯的选择性不对称氢 化, 希望能够高选择性地得到高光学纯度的单一产物. 1991 年 Saburi 等 ${ }^{[14 a, 14 b]}$ 最早研究了 Ru-BINAP 体系对 $\beta, \delta$-二羰基羧酸酯的不对称氢化. 他们的结论认为: 3 -位 羰基首先被氢化, 然后 5-位羰基再被氢化, 但是中间产 物很难被分离出来, 最终得到的是以反式为主的 $\beta, \delta$ 二 羟基羧酸酯, 但是对映选择性和非对映选择性不理想 (Scheme 4). Mortreux 等 ${ }^{[14 c]}$ 在这类底物的氢化过程中检 测到了 $\mathrm{C}(3)$-羟基中间体而没有 $\mathrm{C}(5)$-羊基中间体，这证 实了 3-位羰基首先被还原. 他们以 $97 \%$ 的产率分离得到 了 $\mathrm{C}(3)$-羰基还原产物, 但是 $e e$ 最高只有 78\%; 接下来 使用 (R)-BINAP 作为配体(Eq. 5), 对 66\% ee 的(R)-5-羊圣 基-3-羰基已酸甲酯氢化, 虽然得到了 $90 \%$ 的顺式产物, 主产物 $e e$ 值只有 $75 \%$. 这主要是因为原料的 $e e$ 值不高. 2000 年, Carpentier 小组 ${ }^{[144]}$ 报道了 $\mathrm{Ru}(\mathrm{Ph}, \mathrm{Ph}$-oxo-ProNOP)催化体系, 在最优化的条件下, 一步还原得到了 $96 \%$ 的顺式产物，但是主产物基本上没有立体选择性 (5\% ee, Eq. 6).
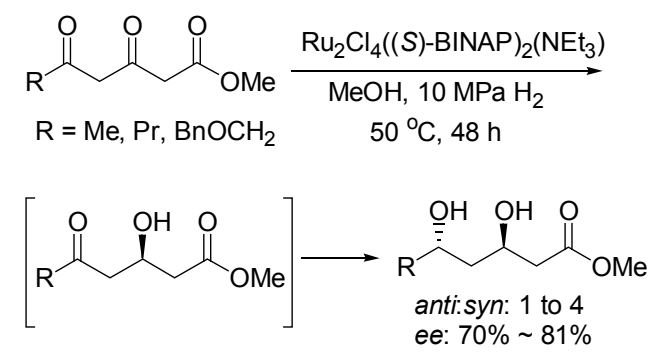

Scheme 4
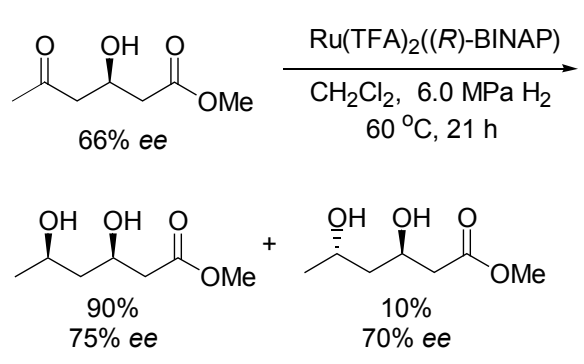

20 年来, 这类反应物的不对称氢化没有取得更多 进展, 主要原因就是多羰基的存在导致竞争配位关系十 分复杂, 使得区域和立体选择性的控制变得十分困难. 我们之前发现 $\beta$-酩酸酯和 $\beta$-酩酰胺在 THF 中的反应活 性有着巨大差别, 通过以往的报道发现, 乙酰丙酮和乙 酰乙酸乙酯的反应活性极为接近(在醇类溶剂中), 我们 猜想在 THF 中它们的活性应该同样很低. 这一猜测得
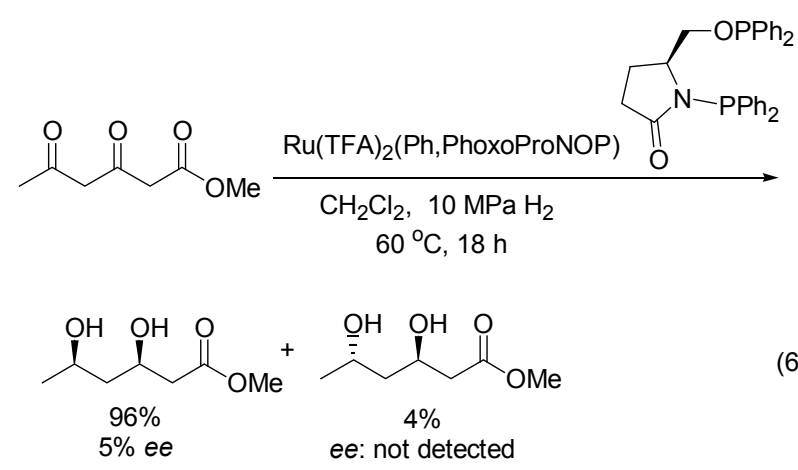

到了验的验证之后, 我们合成了 $18 \mathrm{a}(\mathrm{R}=\mathrm{Me})$, 在 $\mathrm{Ru}-$ SunPhos-THF 体系下氢化, 以接近 $100 \%$ 的收率得到了 3-位氢化的产物, 而且 $e e$ 值高达 96.8\% (Eq. 7). 通过对 底物的扩展, 实现了一系列 3,5-二氧代羧酸酰胺的不对 称氢化 ${ }^{[24]}$. 这种同时兼顾区域和立体选择性的还原之 前还未有报道.

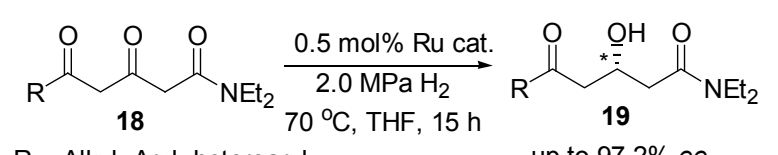

$\mathrm{R}=$ Alkyl, Aryl, heteroaryl up to $97.2 \%$ ee

同时我们 ${ }^{[25]}$ 也发展了用非手性催化剂 $\mathrm{RuCl}_{2}\left(\mathrm{PPh}_{3}\right)_{3}$ 选择性氢化一系列 3,5-二氧代羧酸衍生物的方法, 取得 了很好的 $\mathrm{C}(3)$-羰基选择性, 普通的还原方法目前还无 法实现这种选择性的化学还原(Eq. 8).

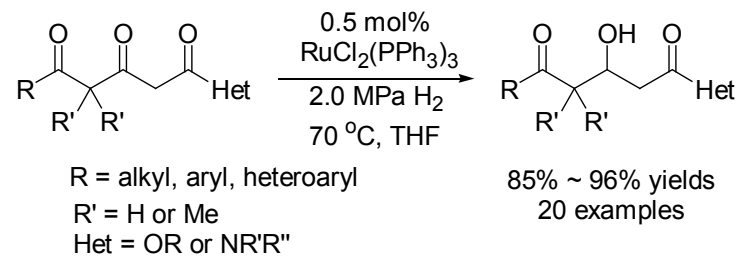

\section{5 总结与展望}

我们课题组近些年在系统研究各种单官能团不对 称氢化反应的基础上，对各种杂原子和羧酸衍生物羰基 在不对称氢化反应中的配位导向能力有了较为深刻的 认识，在多官能羰基酮的不对称氢化方面取得了一些重 要进展. 我们认为, 外来的竞争配位性配体(或溶剂)能 够起到调控催化剂金属中心与底物中不同的导向基团 的竞争配位关系的作用，这对于反应选择性的控制有着 积极作用. 此外, 对于多羰基酮酸衍生物而言, 氢化反 应的机理还有待阐明，目前还没有充足的证据表明是通 过酮式直接氢化还是经过烯醇式的烯烃氢化. 更多深入 的研究正在进行中, 我们希望能够通过涉及机理的研究 来解决更复杂的多官能团羰基的不对称氢化问题, 特别 是希望在区域选择性、对映选择性和非对映选择性方面 


\section{同时取得较大突破.}

\section{References}

[1] (a) Kitamura, M.; Noyori, R. In Ruthenium in Organic Synthesis, Ed.: Murahashi, S.-I., Wiley-VCH, Weinheim, 2004, p. 3.

(b) Noyori, R. Asymmetric Catalysis in Organic Synthesis, Wiley, New York, 1994, p. 17.

(c) de Vries, J. G.; Elsevier, C. J. The Handbook of Homogeneous Hydrogenation, Wiley-VCH, Weinheim, 2007.

[2] Saito, T.; Yokozawa, T.; Ishizaki, T.; Moroi, T.; Sayo, N.; Miura, T.; Kumobayashi, H. Adv. Synth. Catal. 2001, 343, 264.

[3] Tang, W.; Zhang, X. Chem. Rev. 2003, 103, 3029.

[4] Noyori, R.; Ohkuma, T. Angew. Chem., Int. Ed. 2001, 40, 40.

[5] Noyori, R. Asymmetric Catalysis in Organic Synthesis, John Wiley \& Sons, New York, 1994

[6] Sun, Y.; Wan, X.; Guo, M.; Wang, D.; Dong, X.; Pan, Y.; Zhang, Z. Tetrahedron: Asymmetry 2004, 15, 2185.

[7] (a) Sun, Y.; Wan, X.; Guo, M.; Wang, D.; Dong, X.; Pan, Y.; Zhang, Z. Tetrahedron: Asymmetry 2004, 15, 2185.

(b) Wan, X.; Sun, Y.; Luo, Y.; Li, D.; Zhang, Z. J. Org. Chem. 2005, 70, 1070 .

(c) Sun, Y.; Wan, X.; Wang, J.; Meng, Q.; Zhang, H.; Jiang, L.; Zhang, Z. Org. Lett. 2005, 7, 5425.

(d) Wan, X.; Meng, Q.; Zhang, H.; Sun, Y.; Fan, W.; Zhang, Z. Org. Lett. 2007, 9, 5613 .

(e) Meng, Q.; Sun, Y.; Ratovelomanana-Vidal, V.; Genêt, J. P.; Zhang, Z. J. Org. Chem. 2008, 73, 3842.

(f) Tao, X.; Li, W.; Ma, X.; Li, X.; Fan, W.; Xie, X.; Ayad, T.; Ratovelomanana-Vidal, V.; Zhang, Z. J. Org. Chem. 2011, 77, 612. (g) Xu, H.; Meng, Q.; Zhang, Z. Chin. J. Chem. 2008, 26, 1656.

[8] (a) Herold, P.; Indolese, A. F.; Studer, M.; Jalett, H. P.; Siegrist, U.; Blaser, H. U. Tetrahedron 2000, 56, 6497.

(b) Blaser, H. U.; Eissen, M.; Fauquex, P. F.; Hungerbühler, K.; Schmidt, E.; Sedelmier, G.; Studer, M. In Asymmetric Catalysis on Industrial Scale: Challenges, Approaches and Solutions, Eds.: Blaser, H. U., Schmidt, E., Wiley-VCH, Weinheilm, 2004, p. 91.

[9] Meng, Q.; Zhu, L.; Zhang, Z. J. Org. Chem. 2008, 73, 7209.

[10] (a) Zhu, L.; Chen, H.; Meng, Q.; Fan, W.; Xie, X.; Zhang, Z. Tetrahedron 2011, 67, 6186.

(b) Zhu, L.; Meng, Q.; Fan, W.; Xie, X.; Zhang, Z. J. Org. Chem. 2010, 75, 6027.

[11] Fan, W.; Li, W.; Ma, X.; Tao, X.; Li, X.; Yao, Y.; Xie, X.; Zhang, Z. J. Org. Chem. 2011, 76, 9444.

[12] (a) Andrushko, N.; Andrushko, V.; Tararov, V.; Korostylev, A.; König, G.; Börner, A. Chirality 2010, 22, 534.

(b) Ager, D. J.; De Vries, A. H. M.; De Vries, J. G. Chem. Soc. Rev. 2012, 41, 3340.

[13] Yao, Y.; Fan, W.; Li, W.; Ma, X.; Zhu, L.; Xie, X.; Zhang, Z. J. Org. Chem. 2011, 76, 2807.
[14] (a) Shao, L.; Seki, T.; Kawano, H.; Saburi, M. Tetrahedron Lett. 1991, 32, 7699.

(b) Shao, L.; Kawano, H.; Saburi, M.; Uchida, Y. Tetrahedron 1993, 49, 1997.

(c) Blandin, V.; Carpentier, J.-F.; Mortreux, A. Eur. J. Org. Chem. 1999, 3421 .

(d) Blandin, V.; Carpentier, J.-F.; Mortreux, A. Eur. J. Org. Chem. 1999, 1787.

(e) Blandin, V.; Carpentier, J.-F.; Mortreux, A. New J. Chem. 2000, 24,309

[15] Fan, W.; Li, W.; Ma, X.; Tao, X.; Li, X.; Yao, Y.; Xie, X.; Zhang, Z. Chem. Commun. 2012, 48, 4247.

[16] (a) Hiersemann, M.; Nubbemeyer, U. The Claisen Rearrangement: Methods and Applications, Wiley-VCH, Weinheim, 2007.

(b) Wipf, P. In In Comprehensive Organic Synthesis, Vol. 5, Eds.: Trost, B.; Fleming, I.; Paquette, L., Pergamon, Kidlington, Oxford, 1991, p. 827

[17] Stork, G.; Schoofs, A. R. J. Am. Chem. Soc. 1979, 101, 5081.

[18] (a) Yamaguchi, S.; Mosher, H. S. J. Org. Chem. 1973, 38, 1870.

(b) Noyori, R.; Tomino, I.; Tanimoto, Y.; Nishizawa, M. J. Am. Chem. Soc. 1984, 106, 6709.

(c) Terashima, S.; Tanno, N.; Koga, K. J. Chem. Soc., Chem. Commun. 1980, 1026.

[19] (a) Brown, H. C.; Ramachandran, P. V. Acc. Chem. Res. 1992, 25, 16.

(b) Corey, E. J.; Helal, C. J. Angew. Chem., Int. Ed. 1998, 37, 1986.

[20] (a) Ohkuma, T.; Ooka, H.; Ikariya, T.; Noyori, R. J. Am. Chem. Soc. 1995, 117, 10417.

(b) Ohkuma, T.; Koizumi, M.; Doucet, H.; Pham, T.; Kozawa, M.; Murata, K.; Katayama, E.; Yokozawa, T.; Ikariya, T.; Noyori, R. J. Am. Chem. Soc. 1998, 120, 13529.

(c) Arai, N.; Azuma, K.; Nii, N.; Ohkuma, T. Angew. Chem., Int. Ed. 2008, 47, 7457.

[21] (a) Ohkuma, T.; Ooka, H.; Hashiguchi, S.; Ikariya, T.; Noyori, R. $J$. Am. Chem. Soc. 1995, 117, 2675.

(b) Reiff, E. A.; Nair, S. K.; Narayan Reddy, B. S.; Inagaki, J.; Henri, J. T.; Greiner, J. F.; Georg, G. I. Tetrahedron Lett. 2004, 45, 5845.

[22] Ma, X.; Li, W.; Li, X.; Tao, X.; Fan, W.; Xie, X.; RatovelomananaVidal, V.; Ayad, T.; Zhang, Z. Chem. Commun. 2012, 48, 5352.

[23] Li, W.; Ma, X.; Fan, W.; Tao, X.; Li, X.; Xie, X.; Zhang, Z. Org. Lett. 2011, 13, 3876.

[24] Li, W.; Fan, W.; Ma, X.; Tao, X.; Li, X. Zhang, Z. In 7th Chinese Chemical Society National Organic Chemistry Conference, Nanjing, 2011, p. 35 (in Chinese).

(李万方，范为正，马欣，陶晓明，李晓明，张兆国，第七届全国 有机化学学术会议, 南京, 2011, p. 35).

[25] Li, W.; Xie, X.; Tao, X.; Ma, X.; Fan, W.; Li, X.; Zhang, Z. RSC Adv. 2012, 2, 3214. 